\title{
Simultaneous training on two hippocampus-dependent tasks facilitates acquisition of trace eyeblink conditioning
}

\author{
Amy G. Kuo, ${ }^{1,3}$ Grace Lee, ${ }^{2}$ and John F. Disterhoft \\ Department of Physiology and Institute for Neuroscience, Feinberg School of Medicine, Northwestern University, \\ Chicago, Illinois 60611, USA
}

\begin{abstract}
A common cellular alteration, reduced post-burst afterhyperpolarization (AHP) in CAl neurons, is associated with acquisition of the hippocampus-dependent tasks trace eyeblink conditioning and the Morris water maze. As a similar increase in excitability is correlated with these two learning paradigms, we sought to determine the interactive behavioral effects of training animals on both tasks by using either a consecutive or simultaneous training design. In the consecutive design, animals were trained first on either the trace eyeblink conditioning task for six sessions, followed by training on the water maze task for six sessions, or vice versa. The simultaneous design consisted of six or 11 training days; animals received one session/day of both trace eyeblink conditioning and water maze training. Separate groups were used for consecutive and simultaneous training. Animals trained on both tasks simultaneously were significantly facilitated in their ability to acquire the trace eyeblink conditioning task; no effect of simultaneous training was seen on the water maze task. No effect was seen on acquisition for either task when using the consecutive training design. Taken together, these findings provide insight into how the hippocampus processes information when animals learn multiple hippocampus-dependent tasks.
\end{abstract}

The importance of the hippocampus in learning is well established (Berger et al. 1976; Berger and Thompson 1978; Disterhoft et al. 1999). A number of studies have demonstrated the necessity of the hippocampus in learning spatial and nonspatial tasks, as well as those requiring the association of temporally remote events (Morris et al. 1982; Moyer Jr. et al. 1990; Davidson and Jarrard 1993; McEchron et al. 1998; Bannerman et al. 1999; Weiss et al. 1999a). Two such tasks are trace eyeblink conditioning (EBC) and Morris water maze (WMZ).

A common cellular mechanism that underlies learning, increased membrane excitability, has been observed by several investigators. This mechanism is conserved across species, tasks, and brain regions. Trains of action potentials in both hippocampal and cortical neurons are followed by a prolonged post-burst afterhyperpolarization (AHP), which decreases neuronal excitability and inhibits further firing to a sustained depolarizing input (Schwindt et al. 1988; Storm 1990). A reduction in the AHP occurred in both rat and rabbit CA1 pyramidal neurons after acquisition of trace EBC (Moyer Jr. et al. 1996; Kuo et al. 2004). This reduction also occurred in rat CA1 pyramidal neurons after acquisition of another type of hippocampus-dependent task, the spatial Morris WMZ (Oh et al. 2003). Alterations in the AHP with learning have also been observed in rat piriform cortical neurons after acquisition of an odor discrimination task (Saar et al. 1998). In both hippocampus and piriform cortex, the reduced AHP followed a similar time course after learning, with a maximal reduction $24 \mathrm{~h}$ after task acquisition and a return to baseline within 7 d of acquisition (Moyer Jr. et al. 1996; Saar et al. 1998).

Present addresses: ${ }^{1}$ Molecular and Behavioral Neuroscience Institute, University of Michigan, Ann Arbor, Michigan 48109-0669, USA; 2University of Wisconsin, Madison, Wisconsin 53706, USA.

${ }^{3}$ Corresponding author.

E-mail agkuo@umich.edu; fax (734) 936-2690.

Article and publication are at http://www.learnmem.org/cgi/doi/10.1101/ Im.98406.
In addition to learning-related alterations of intrinsic excitability, synaptic changes have been shown to accompany hippocampus-dependent learning. Geinisman et al. (2000) have shown that $24 \mathrm{~h}$ following trace EBC acquisition, an increase in the postsynaptic density (PSD) area of nonperforated axospinous synapses is seen in CA1 pyramidal neurons. In addition, PSD size of CA1 pyramidal perforated axospinous synapses has been correlated with WMZ learning ability in aging rats (Nicholson et al. 2004). Enhanced synaptic transmission and responsiveness in CA1 pyramidal neurons have also been shown to accompany EBC acquisition. Subsequent to delay EBC acquisition, a learning-specific increase in summating EPSPs was seen in CA1 pyramidal neurons (LoTurco et al. 1988). In addition, Schaffer collateral stimulation resulted in greater evoked field potentials in CA1 pyramidal neurons from trace EBC animals, compared with neurons from pseudoconditioned animals (Power et al. 1997). There is also evidence for long-term potentiation (LTP) as a cellular phenomenon associated with hippocampal learning. Results from both EBC and WMZ experiments indicate that alterations in synaptic weights as a consequence of LTP is one way that the hippocampus may store information during acquisition of these tasks (Berger 1984; Castro et al. 1989; Moser et al. 1998; Christian and Thompson 2003).

Although enhanced excitability and synaptic transmission have been reliably shown to be cellular mechanisms of learning after single task acquisition, it is not known how these changes will impact learning a second hippocampus-dependent task. This question could be addressed by requiring animals to acquire both tasks in either a consecutive or simultaneous fashion. Consecutive training would require the animal to learn a second task after acquisition of an initial task was complete. Acquisition of this second task would presumably occur when cellular alterations are still present in CA1 neurons due to acquisition of the first task, allowing us to examine their effect on acquisition of the second. Simultaneous training would require the animal to ac- 
quire two hippocampus-dependent tasks in parallel, thereby allowing us to examine the interaction of the developing cellular changes and the animal's ability to learn.

The findings from these experiments would provide insight into how intrinsic and synaptic cellular alterations impact subsequent or simultaneous learning. Although the likely function of these changes is to promote subsequent learning by "priming" the hippocampal circuit, it is possible that they could interfere with or occlude acquisition of a task that requires further enhancement of these neuronal properties. Results from studies examining the impact of LTP induction on learning lend support to the former outcome. Berger (1984) demonstrated that when LTP was induced in the perforant path-dentate gyrus pathway in vivo, animals receiving highfrequency stimulation of this pathway subsequently learned a discrimination EBC task significantly better than did animals receiving low-frequency stimulation. In addition, in vivo kindling of this pathway in rabbits has been shown to result in enhanced acquisition of the discrimination task (Robinson et al. 1989; although see Rioux and Robinson 1995). Spatial learning ability has also been shown to be influenced by prior LTP induction (Castro et al. 1989; Barnes et al. 1994; Moser et al. 1998). In these studies, saturating LTP of the perforant path-dentate gyrus synapses was found to impair subsequent spatial learning. Thus, the data from these studies suggest that, depending on the level of LTP induction, prior synaptic change has a profound effect on subsequent learning.

To date, no study has determined the effect of training an animal on two hippocampus-dependent tasks in close temporal proximity. To examine this effect, we trained animals on both trace EBC and WMZ by using either a consecutive or simultaneous training design. Surprisingly, consecutive training had no effect on acquisition of either task. However, simultaneous training on trace $\mathrm{EBC}$ and WMZ tasks significantly enhanced rats' ability to acquire the trace eyeblink conditioning task as compared to controls.

\section{Results}

\section{Consecutive training does not enhance either trace EBC acquisition or WMZ learning}

When animals were trained on both the trace EBC and WMZ tasks consecutively, no enhancement of learning was seen for either task. Consecutive training pro-

B

C course of training. ceeded as shown in Figure 1A. Animals trained first on trace EBC followed by WMZ training (EBC $\rightarrow \mathrm{WMZ}, n=9$ ) were able to acquire the trace $\mathrm{EBC}$ task as well as were animals trained first on $\mathrm{WMZ}$ followed by $\mathrm{EBC}(\mathrm{WMZ} \rightarrow \mathrm{EBC}, n=10)$. A repeatedmeasures ANOVA revealed no effect of training status on $\mathrm{CR}$
A

\section{Consecutive Training}

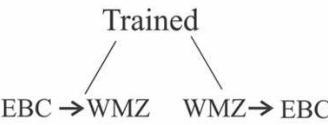

EBC $\rightarrow$ WMZ Training Protocol

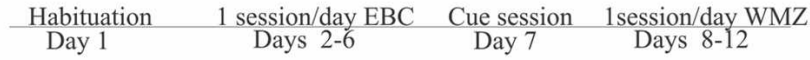

WMZ $\rightarrow$ EBC Training Protocol

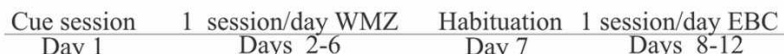

\section{Simultaneous Training}

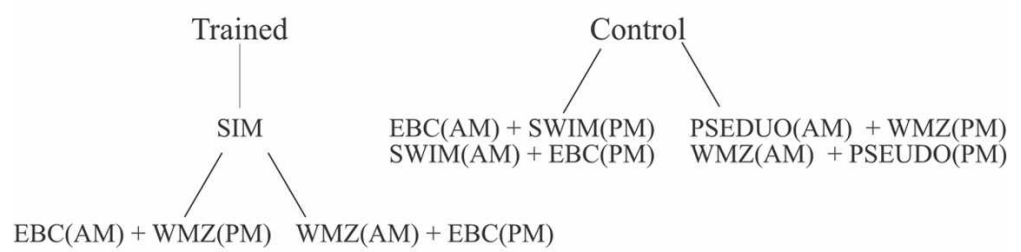

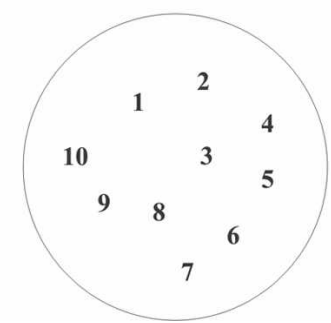

Figure 1. (A) Experimental groups and training timeline for consecutive training. Animals were either trained on trace eyeblink conditioning for $6 \mathrm{~d}$ (one habituation session + five training sessions) followed by WMZ training (one cue session + five training sessions) (EBC $\rightarrow$ WMZ), or trained on WMZ first followed by training on trace eyeblink conditioning (WMZ $\rightarrow E B C)$. (B) Experimental groups and training timeline for simultaneous training. Simultaneously trained (SIM) animals were either trained on trace eyeblink conditioning in the morning followed by WMZ in the afternoon (EBC(AM) + WMZ(PM)) or vice versa $(W M Z(A M)+E B C(P M))$. Control groups were counterbalanced for morning and afternoon training sessions. Control animals were either trained on trace eyeblink conditioning and swam with no platform in the tank (time-yoked to a trained animal; EBC + SWIM) or pseudoconditioned on trace eyeblink conditioning and trained on WMZ (PSEUDO + WMZ). Simultaneous animals received a cue session (WMZ) and a habituation session (eyeblink conditioning) on day 1, followed by five or 10 sessions of trace eyeblink conditioning and WMZ training (one session each task/day). (C) Platform locations in the WMZ tank. The hidden platform was moved to one of 10 random locations over the

\section{Learning \& Memory}


percentage $\left(F_{[1,17]}=2.5, P=0.13\right)$ (Fig. $\left.2 \mathrm{~B}\right)$. Similarly, there was no difference in $\mathrm{WMZ}$ learning between $\mathrm{EBC} \rightarrow \mathrm{WMZ}$ and $\mathrm{WMZ} \rightarrow \mathrm{EBC}$ animals. A repeated-measures ANOVA revealed no significant effect of training status on latency to hidden platform across sessions $\left(F_{[1,17]}=0.02, P=0.089\right)$ (Fig. 2C).

\section{Simultaneous training enhances acquisition of trace EBC but not Morris WMZ}

Simultaneous training proceeded as shown in Figure 1B. When animals were trained on both the trace EBC and WMZ tasks simultaneously, i.e., one training session of each task on each training day, a significant enhancement of trace EBC acquisition

A

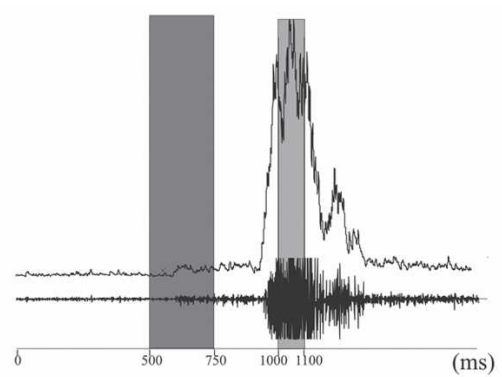

B

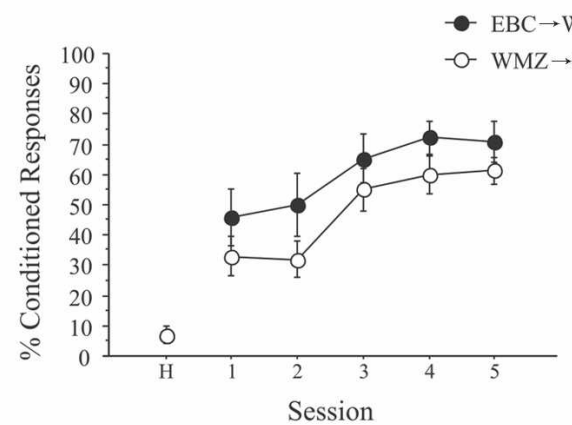

C

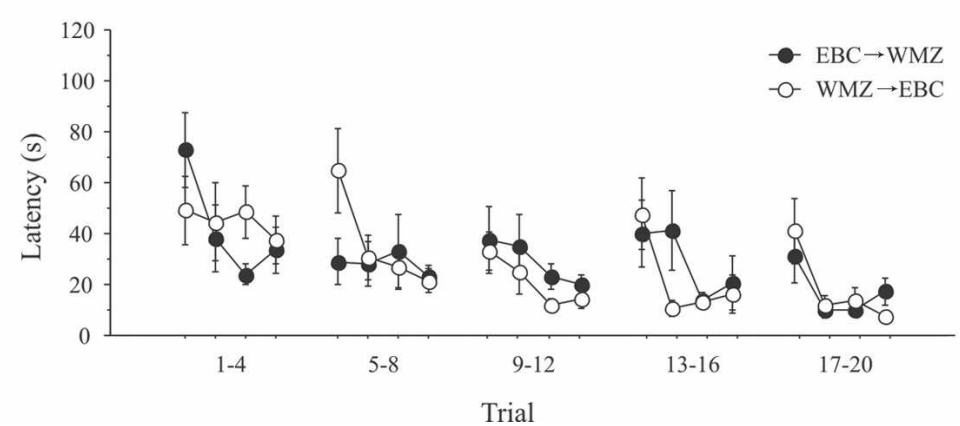

Figure 2. (A) Example of an eyeblink response exhibited by a rat receiving paired presentations of the CS and US (trained). Example taken from session 5, with the animal achieving $82 \%$ CRs on this session. Both raw EMG (bottom) and rectified/integrated EMG (top) responses are shown. Shaded bars indicate the timing of the tone CS (dark gray) and airpuff US (light gray). (B) Acquisition rates of trace eyeblink conditioning for consecutively trained animals. There was no difference between $E B C \rightarrow W M Z$ and $W M Z \rightarrow E B C$ animals for $C R$ percentage over training. (C) Latency to the hidden platform over training trials for consecutively trained animals. There was no difference between $E B C \rightarrow W M Z$ and $\mathrm{WMZ} \rightarrow \mathrm{EBC}$ animals for latency to the platform over training. was seen. However, the converse enhancement of learning was not observed on the WMZ task. An initial group of animals was trained by using this paradigm for five sessions each of trace EBC and WMZ. There was no significant effect of training order on trace EBC task acquisition for simultaneously trained (SIM) animals; animals that were trained on the trace EBC task in the morning, followed by WMZ in the afternoon EBC(AM) + WMZ(PM)) learned the task as well as did animals that were trained on the WMZ task in the morning followed by trace $\mathrm{EBC}$ training in the afternoon (WMZ(AM) + EBC(PM)) $\left(F_{[1,10]}=0.32, P=0.59\right)$. Thus, these two groups were combined for further analyses. As shown in Figure 3A, animals that received training sessions of both trace $\mathrm{EBC}$ and WMZ each day (SIM, $n=12$ ) learned trace EBC significantly better than did animals that were trained on trace EBC and were time-yoked swim controls on the WMZ (EBC + SWIM, $n=7)$. ANOVA revealed a significant effect of training status on CR percentage $\left(F_{[2,24]}=23.52\right.$, $P<0.0001)$. Post hoc LSD tests indicated that SIM animals acquired the trace EBC task significantly better than did EBC + SWIM animals (mean difference $=17.43, P=0.009)$. In addition, post hoc LSD tests indicated that both SIM and EBC + SWIM animals learned the task significantly better than did animals that served as pseudoconditioned controls in the eyeblink task and trained on the WMZ (PSEUDO + WMZ, $n=8$; SIM, PSEUDO + WMZ: mean difference $=40.51, P<0.001 ; \mathrm{EBC}+\mathrm{SWIM}$, PSEUDO + WMZ: mean difference $=23.08, P=0.002$ ) .

There was no effect of simultaneous training on WMZ learning. PSEUDO + WMZ animals were able to acquire this task as well as were SIM animals. ANOVA revealed there was no significant effect of training status on latency to platform across trials $\left(F_{[1,18]}=0.62, P=0.44\right)$ (Fig. $\left.3 \mathrm{~B}\right)$.

The results from the simultaneous training protocol clearly indicated that animals that receive training on two tasks each day over the course of training (simultaneous group) are significantly enhanced in their ability to acquire the trace EBC task compared with animals that learned only one task per day. Given that this experiment used a relatively short training protocol (five training sessions) and an enhancement was still present on the final training session, we extended the number of training sessions to determine if this facilitation of learning was confined to the early stages of trace EBC acquisition or was an enhancement that persisted into the asymptotic phase of learning.

By using a 10-training-session protocol (Fig. 1B), we found that, similar to the five-training-session experiment, SIM animals $(n=9)$ achieved a significantly greater percentage of CRs than 
A

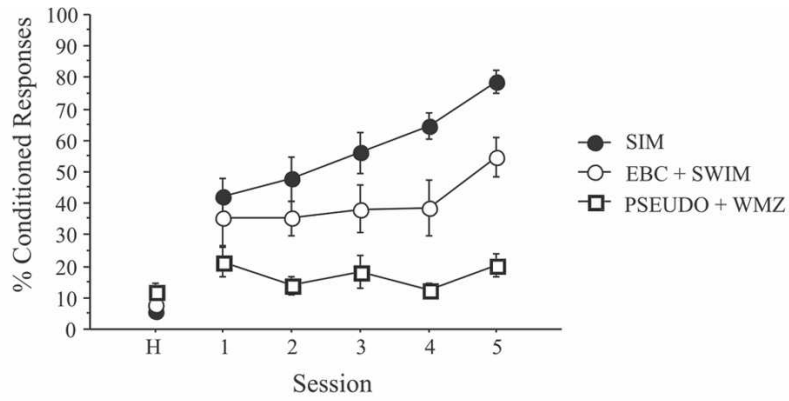

B

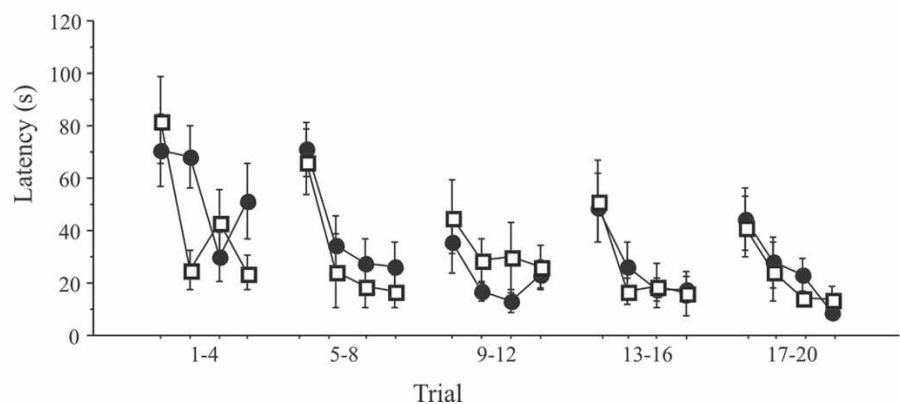

Figure 3. (A) Acquisition rates of trace eyeblink conditioning for five-session simultaneous training protocol. SIM animals achieved a significantly greater percentage of CRs than did EBC + SWIM animals over the five training sessions (Fisher's LSD, $P=0.009$ ). Animals in both SIM and EBC + SWIM groups learned the task significantly better than did PSEUDO + WMZ animals (SIM, PSEDUO + WMZ, $P<0.01$; EBC + SWIM, PSEUDO + WMZ, $P=0.002$ ). (B) Learning curve for WMZ acquisition for the five-session simultaneous training paradigm. There was no difference in latency to the platform between SIM and PSEUDO + WMZ animals.

did EBC + SWIM animals $(n=9)$ (Fig. 4A). Again, there was no effect of training order for SIM animals; there was no difference in $\mathrm{CR}$ percentage over training between $\mathrm{EBC}(\mathrm{AM})+\mathrm{WMZ}(\mathrm{PM})$ and $\mathrm{WMZ}(\mathrm{AM})+\mathrm{EBC}(\mathrm{PM})$ animals $\left(F_{[1,7]}=1.09, P=0.33\right)$, so these two groups were combined for further analyses. A repeatedmeasures ANOVA revealed an effect of training status on CR percentage $\left(F_{[2,25]}=79.68, P<0.0001\right)$. Post hoc LSD tests indicated that SIM animals achieved a significantly higher percentage of CRs compared with EBC + SWIM animals (mean difference $=11.21$, $P=0.02$ ). Post hoc LSD tests also indicated that both SIM and EBC + SWIM animals learned the trace EBC task better than did PSEUDO + WMZ animals $(n=10)$ (SIM, PSEUDO + WMZ, $P<0.0001 ; \mathrm{EBC}+$ SWIM, PSEUDO + WMZ, $P<0.0001)$.

In addition, a further analysis of CR percentage over training trials revealed that this enhancement was restricted to the early stages of learning. ANOVA revealed that SIM animals achieved a significantly greater percentage of CRs over sessions 1 to 6 compared with EBC + SWIM animals $\left(F_{[1,16]}=4.6, P<0.05\right)$, but that for sessions 7 to 10 this enhancement was no longer significant $\left(F_{[1,16]}=2.16, P=0.16\right)$ (Fig. $\left.4 \mathrm{~A}\right)$. As further confirmation of this result, the trace EBC data were also analyzed to determine when rats reached a learning criterion of eight CRs within 10 consecutive trials, an indication of rate of learning. As seen in Figure 4B, an unpaired $t$-test revealed that SIM animals required significantly fewer trials to achieve this criterion than did EBC + SWIM animals $(P<0.05)$. As seen with the fivetraining-session protocol, there was no difference between SIM and PSEUDO + WMZ groups for WMZ learning using a 10session training protocol $\left(F_{[1,17]}=0.62, P=0.44\right)$ (Fig. $4 \mathrm{C}$ ).

A subset of animals was also trained on the nonhippocampus-dependent delay EBC task to further explore whether the enhancement of trace $\mathrm{EBC}$ by simultaneous training on the WMZ task was due to the hippocampal nature of these two tasks. Animals either were trained on both the delay EBC task and the WMZ task (DELAY + WMZ, $n=8$ ) or were time-yoked swim control animals and trained on the delay EBC task (DELAY + SWIM, $n=8$ ) for five training sessions. Training of animals was counterbalanced for time of day. As observed in the previous sets of experiments, no effect of time of day was seen on training for either the experimental or control groups, so all animals within these two groups were combined for further analysis. ANOVA revealed that delay EBC was not enhanced by simultaneous training on the WMZ task on the same day, as there was no difference in CR percentage between DELAY + WMZ and DELAY + SWIM animals $\left.\left(F_{[1.13}\right]=0.007, P=0.93\right)$ (Fig. 4D).

\section{Discussion}

The results of the current study demonstrate that simultaneous training on two hippocampus-dependent tasks, trace $\mathrm{EBC}$ and WMZ, results in a facilitation of the EBC task, with no effect on WMZ learning. This enhancement of the trace EBC task was only seen when animals were trained in a simultaneous fashion; no effect was seen on either trace EBC or WMZ learning when animals were trained on these two tasks consecutively. This facilitation was confined to the early phase of learning and was no longer present after animals reached asymptotic levels of learning. The facilitation of trace $\mathrm{EBC}$ by simultaneous training on WMZ was a learning-specific effect, and not the result of a stress effect, as animals trained on both trace EBC and WMZ learned the EBC task significantly better than did those trained on trace EBC plus the time-yoked swim control procedure. Finally, this facilitation was specific for the hippocampus-dependent form of EBC, as there was no effect of simultaneous WMZ learning on delay conditioning. To date, this is the first examination of the effects of training an animal on two hippocampus-dependent tasks in close temporal proximity.

Prior work by our laboratory indicates that a common cellular mechanism subserves acquisition of both the trace EBC and WMZ tasks. Acquisition of either trace EBC (rats and rabbits) or WMZ (rats) results in a reduction of the post-burst AHP in CA1 pyramidal neurons (Moyer Jr. et al. 1996; Oh et al. 2003; Kuo et al. 2004). In addition, systemic administration of compounds that reduce the AHP result in an enhancement of learning hippocampus-dependent tasks (Kronforst-Collins et al. 1997; Oh et al. 1999; Weiss et al. 2000). Based on these findings, we hypothesized that training rats on two hippocampus-dependent tasks would result in facilitation of the second task. Surprisingly, this was not the case; acquisition of trace EBC had no effect on subsequent WMZ learning, and vice-versa. Although we saw no effect with consecutive training, simultaneous training yielded an interesting pattern of results. The lack of an effect of simultaneous training on WMZ learning was surprising given the significant enhancement of trace EBC by this training paradigm. However, there are several possible explanations for this disparate effect. In addition to a reduction in the AHP, other cellular alterations occur with learning that may account for our results. An increase in summating EPSPs and synaptic transmission has been shown to occur in CA1 pyramidal neurons from trace EBC animals compared with neurons from pseudoconditioned and 
A

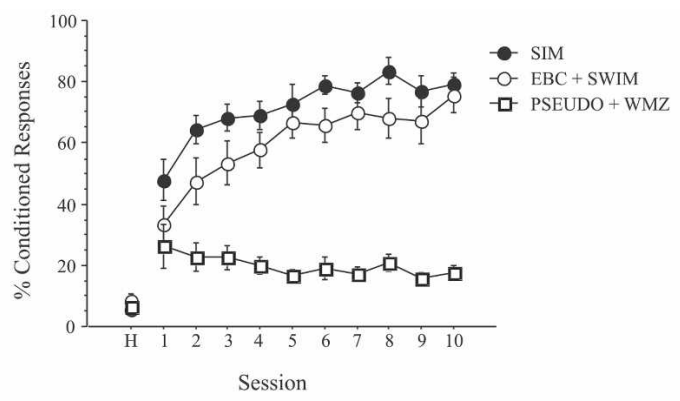

$\mathrm{B}$

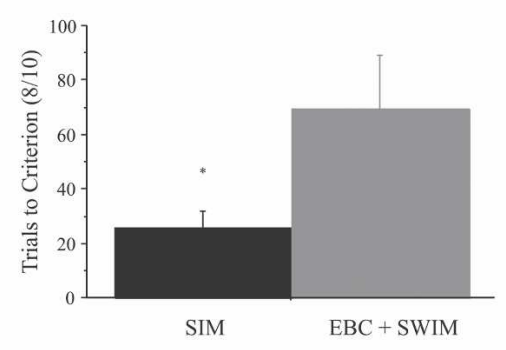

$\mathrm{C}$

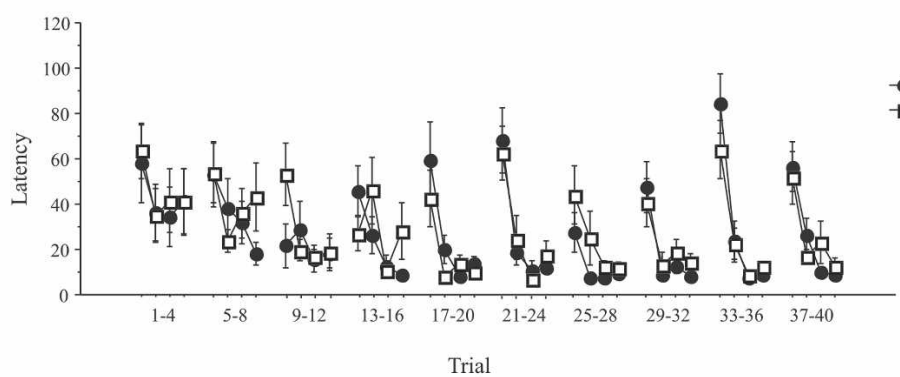

$\mathrm{D}$

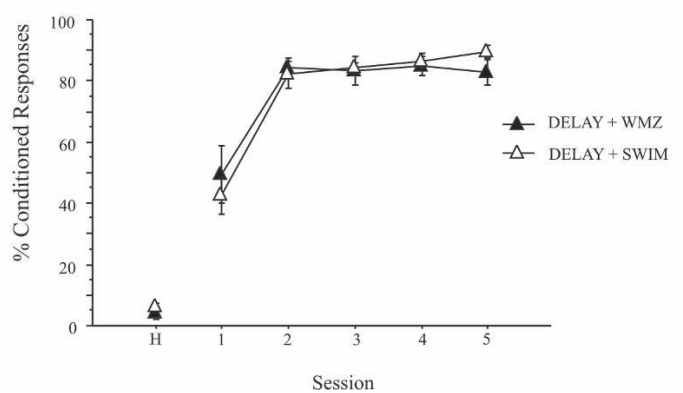

Figure 4. (A) Acquisition rates of trace eyeblink conditioning for 10 -session simultaneous training paradigm. SIM animals achieved a significantly greater percentage of CRs over the 10 training sessions compared with $E B C+$ SWIM animals (Fisher's $L S D, P=0.02$ ). This enhancement of $C R$ percentage was confined to sessions 1 to $6\left(F_{[1,16]}=4.6, P<0.05\right)$; there was not a significant difference in CR percentage between SIM and EBC + SWIM animals for trials 7 to $10\left(F_{[1,16]}=2.16, P=0.16\right)$. Both SIM and $E B C+$ SWIM animals learned the trace eyeblink conditioning task significantly better than did PSEUDO + WMZ animals (SIM, PSEUDO + WMZ, P<0.0001; EBC + SWIM, PSEUDO + WMZ, $P<0.0001)$. (B) Number of training trials required for animals to reach a criterion of eight of $10 \mathrm{CRs}$. SIM animals reached an eight of $10 \mathrm{CR}$ criterion in significantly fewer trials than did EBC + SWIM animals. (C) Learning curve for WMZ acquisition for the 10-session simultaneous training paradigm. There was no difference in latency to the platform between SIM and PSEUDO + WMZ animals. (D). Acquisition rates of delay eyeblink conditioning. There was no difference in CR percentage over training between DELAY + WMZ and DELAY + SWIM animals. ${ }^{*} P<0.05$, unpaired $t$-test. synaptic transmission and synaptic responsiveness due to acquisition of one task could render the hippocampal circuit more excitable to subsequent input, such as from a second task. In addition, alterations in LTP have been shown to be important for learning hippocampusdependent tasks. Increases in synaptic strength as a consequence of LTP have been suggested to underlie increases in in vivo firing of CA1 pyramidal neurons during EBC acquisition, as well as spatial learning ability (Castro et al. 1989; Barnes et al. 1994; Moser et al. 1998; Christian and Thompson 2003). In support of this idea, Berger (1984) showed that animals receiving high-frequency stimulation of the perforant-path dentate gyrus pathway showed enhanced subsequent discrimination EBC compared with animals receiving lowfrequency stimulation of this pathway. In addition to bolstering support for LTP as a cellular analog of learning, these data also suggest that when the hippocampal circuit enters a more excitable state as a consequence of LTP induction, subsequent learning is enhanced. In addition, Robinson et al. (1989) showed that kindling, of which LTP is a component, enhanced rabbits' ability to acquire the discrimination EBC task. The results of the current study are in line with these findings; learning of one task enhances acquisition of a second task, presumably via an alteration of a common cellular mechanism(s). LTP induction has also been shown to have effects on spatial learning. Several groups have shown that when the perforant pathdentate synapses are saturated, subsequent spatial learning is impaired (McNaughton et al. 1986; Castro et al. 1989; Barnes et al. 1994; Moser et al. 1998). Barnes et al. (1994) have shown that there is a correlation between the amount of LTP induced at these synapses and learning ability. In addition, Castro et al. (1989) showed that spatial learning remained impaired in animals that received saturating stimulation of the perforant-dentate pathway until LTP had decayed. Once LTP returned to baseline levels in these animals, they were able to acquire the spatial task as well as did controls. These data demonstrate that depending on the level of LTP induction, subsequent spatial and temporal associative learning is profoundly influenced, and alterations in this plasticity as a consequence of learning may account for the results reported here.

naive animals (Weisz et al. 1984; LoTurco et al. 1988; Power et al. 1997). Increases in synaptic transmission have also been shown to occur in the hippocampus after other types of learning (Skelton et al. 1987; Moser et al. 1994). Either (or both) an increase in
In light of the LTP and kindling learning studies, it is surprising that we did not see an effect with consecutive training on trace EBC acquisition, but it is possible that maximal synaptic or LTP-like changes occurring with WMZ learning happen during 
an early phase of task acquisition. In support of this idea is the fact that we saw an effect on trace EBC with simultaneous WMZ learning. It is possible that synaptic changes reach a maximal level early in training, e.g., after two sessions, resulting in an enhancement on trace EBC as observed here. The fact that we saw an enhancement on an early phase of EBC also supports this. A difference in the time course of synaptic alterations between trace $\mathrm{EBC}$ and WMZ may account for the lack of an effect of simultaneous training on WMZ task acquisition. It is possible that synaptic changes occurring as a consequence of trace EBC occur later in training, thus by the time these changes have occurred, animals have reached asymptotic levels of learning on the WMZ. A detailed study of how these synaptic changes occur over the course of learning (as opposed to after learning has occurred) will clarify how synaptic changes resulting from multiple task acquisition interact.

In addition, it is possible that the consolidation demands of trace $\mathrm{EBC}$ and the water mask task differ, with trace EBC consolidation benefiting from training on the WMZ task between sessions, but no such effect on WMZ learning. Such a scenario would also explain why a facilitation of trace $\mathrm{EBC}$ was only seen when animals were also trained on the WMZ task between EBC sessions (simultaneous training), as opposed to completing training on the WMZ task prior to $\mathrm{EBC}$ training (consecutive training).

The results of this study provide insight into how the hippocampus processes information when animals learn multiple hippocampus-dependent tasks. Although trace EBC and WMZ learning result in some similar cellular changes in CA1 pyramidal neurons, it is clear that a complex interaction occurs between these neurons during simultaneous training resulting in an enhancement of trace EBC, but not WMZ learning. Future work will focus on elucidating the mechanism underlying this disparate effect of simultaneous training.

\section{Materials and Methods}

\section{Subjects}

Four-month-old male F1 hybrid Fischer- $344 \times$ Brown Norway rats were used as subjects. Rats were obtained from the National Institute on Aging and were housed four per cage in a climatecontrolled room on a 12-h light/12-h dark cycle with ad libitum access to food and water. All procedures were done following guidelines maintained by the National Institutes of Health and with approval of the Northwestern University Animal Care and Use Committee.

\section{Surgery}

Procedures of EBC as modified for the rat by Weiss et al. (1999b) were employed in this study. Animals were anesthetized with a combination of xylazine $(13 \mathrm{mg} / \mathrm{kg}$, i.p.) and ketamine $(87 \mathrm{mg} /$ $\mathrm{kg}$, i.p.) and placed in a stereotaxic device. An incision was made on the top of the skull, allowing for retraction of the periosteum. Six bilateral holes were drilled into the skull for insertion of stainless steel screws. A strip connector with two Teflon-coated stainless steel wires was then placed on the skull. EMG activity was recorded from the orbicularis oculi muscle via these wires that were inserted underneath the skin until they penetrated the skin of the upper eyelid of the right eye. A tether holding a connector for relaying EMG activity and a tube for airpuff delivery was attached to the strip connector. Dental cement was then placed around the connector and over the screws until the connector was firmly in place. Following surgery, animals were placed on a heating pad until fully mobile, returned to their home cages, and given $5 \mathrm{~d}$ minimum to recover before beginning behavioral training.

\section{Eyeblink Conditioning}

Prior to the first training session, rats were placed in the training environment for a habituation period lasting the duration of a training session. During this habituation period, neither the conditioned stimulus (CS) nor the unconditioned stimulus (US) were presented. The training environment consisted of a Plexiglas cage placed in a sound attenuating chamber, allowing rats to move freely during training. Training sessions consisted of 30 paired presentations per session of a CS (a 250-msec, $8-\mathrm{kHz}, 85-\mathrm{dB}$ free field tone) and a US (a 100-msec, 4.5-psi corneal airpuff), with an intertrial interval (ITI) of 20-40 sec, with a 30-sec average. For trace EBC, the tone and the airpuff were separated by a $250-$ msec blank trace interval. During delay conditioning, the tone was presented immediately before the airpuff. Pseudoconditioned animals served as behavioral controls. Pseudoconditioning trials consisted of explicitly unpaired presentations of either tone (CS) alone or airpuff (US) alone trials, with a random ITI of $10-20 \mathrm{msec}$ with an average of 15 sec. A Power Macintosh computer running customized LabView software controlled stimulus presentations and data collection, analysis, and storage. The EMG output was amplified $(5000 \times)$ and filtered to pass $100-5000 \mathrm{~Hz}$. This signal was digitized at $3 \mathrm{kHz}$ and stored on the computer. Data were analyzed by using ANOVA and post hoc least significant difference (LSD) tests using Statview software. A conditioned response was one that occurred at $200 \mathrm{msec}$ prior to US onset and was greater than the mean baseline amplitude plus four times the standard deviation of the baseline. Figure $2 \mathrm{~A}$ shows a typical response from a trained animal. Both raw and integrated EMG activity is shown.

\section{Morris WMZ}

Training procedures and performance evaluation are adapted from those designed by Steele and Morris (1999) and von Listow Roloff and colleagues (2002). Prior to the first training session, cue learning was measured. A black platform extending $2 \mathrm{~cm}$ above water level was used for these trials. For each trial the platform was randomly moved, and rats were placed in the tank at a different start position. Rats received four cue trials. Cue learning served as a control procedure for place learning to ensure that the rats did not suffer from sensory, motor, or motivational deficits. Rats were tested for place learning on subsequent sessions; each session consisted of four trials/session. An ITI of 60 sec was used. Prior to the first training trial on day 1 , rats were placed on the platform and allowed to remain there for $30 \mathrm{sec}$ to familiarize themselves with its location. The platform was located in a novel location each session, remaining constant over trials for a given session. Figure $1 \mathrm{C}$ shows the 10 random platform locations in the tank. A matching to sample version of the WMZ task was employed for this study because of the increased difficulty of this task over a standard reference memory WMZ (Harker and Whishaw 2004). On each trial rats were placed in the water, with their heads facing toward the tank wall, at one of four equally spaced positions around the tank (e.g., north, south, east, west). Rats started from a different position each trial, and were allowed to swim until reaching the platform and climbing on top, or $120 \mathrm{sec}$ had expired. Rats that did not find the platform within $120 \mathrm{sec}$ were hand-guided to it. Rats remained on the platform for $15 \mathrm{sec}$ before being removed. Time-yoked swim animals served as controls. Tank water was made opaque with a powdered, nontoxic white paint, and color was kept consistent throughout the training period. Daily readings were taken to verify that tank water was at $25 \pm 1^{\circ} \mathrm{C}$ before animals were run. During all trials, rats' performance was videotaped by a FC-82B video camera and wide-angle camera lens mounted above the center of the pool. The animal's location in the tank was digitized with a VP200 tracker and data was collected by HVS Water for Windows software supplied by HVS Image. The tank was surrounded by curtains with detailed posters attached to them serving as the extramaze cues. The experimenter remained outside the curtains during trials and entered at the end of a trial to retrieve the animals. Placement of the extramaze cues remained consistent from day to day.

Place learning was assessed by analyzing the latency to the platform. 


\section{Training Design}

Training proceeded as outlined in Figure 1. Consecutive training consisted of 12 training days total (Fig. 1A). Groups were counterbalanced so half of the animals were trained first on trace EBC followed by WMZ training (EBC $\rightarrow \mathrm{WMZ}$ ), the other half were trained on WMZ first followed by trace EBC (WMZ $\rightarrow$ EBC). Day 1 of training consisted of either cue training or habituation, days 2 to 6 consisted of two sessions/day of either trace EBC or WMZ training. Daily sessions were separated by $4 \mathrm{~h}$. On the day immediately following the final training session for task one, training on task two began.

Simultaneous training was composed of either six or 11 training days total (Fig. 1B). Trained animals (SIM) were counterbalanced so that half the animals received trace $\mathrm{EBC}$ in the morning, with WMZ training in the afternoon $(\mathrm{EBC}(\mathrm{AM})+\mathrm{WMZ}(\mathrm{PM}))$, and the other half received WMZ training in the morning, trace EBC in the afternoon (WMZ(AM) $+\mathrm{EBC}(\mathrm{PM}))$. Control animals were composed of animals that were pseudoconditioned and trained on the WMZ task $($ PSEUDO(AM) + WMZ(PM); WMZ(AM) + PSEUDO(PM)) and time-yoked swim controls trained on trace EBC (SWIM(AM) $+\mathrm{EBC}(\mathrm{PM}) ; \mathrm{EBC}(\mathrm{AM})+\mathrm{SWIM}(\mathrm{PM}))$. Day 1 of training consisted of a habituation and cue session. Days 2 to 6 or days 2 to 11 consisted of one session/day each trace EBC and WMZ training. Daily sessions were separated by $4 \mathrm{~h}$.

\section{Acknowledgments}

This work was supported by National Institutes of Health Grants AG08796 (J.F.D.) and MH12761 (A.G.K.).

\section{References}

Bannerman, D.M., Yee, B.K., Good, M.A., Heupel, M.J., Iversen, S.D., and Rawlins, J.N. 1999. Double dissociation of function within the hippocampus: A comparison of dorsal, ventral, and complete hippocampal cytotoxic lesions. Behav. Neurosci. 113: 1170-1188.

Barnes, C.A., Jung, M.W., McNaughton, B.L., Korol, D.L., Andreasson, K., and Worley, P.F. 1994. LTP saturation and spatial learning disruption: Effects of task variables and saturation levels. J. Neurosci. 14: $5793-5806$.

Berger, T.W. 1984. Long-term potentiation of hippocampal synaptic transmission affects rate of behavioral learning. Science 224: 627-630.

Berger, T.W. and Thompson, R.F. 1978. Neuronal plasticity in the limbic system during classical conditioning of the rabbit nictitating membrane response, II: Septum and mammillary bodies. Brain Res. 156: $293-314$.

Berger, T.W., Alger, B., and Thompson, R.F. 1976. Neuronal substrate of classical conditioning in the hippocampus. Science 192: 483-485.

Castro, C.A., Silbert, L.H., McNaughton, B.L., and Barnes, C.A. 1989. Recovery of spatial learning deficits after decay of electrically induced synaptic enhancement in the hippocampus. Nature 342: 545-548.

Christian, K.M. and Thompson, R.F. 2003. Neural substrates of eyeblink conditioning: Acquisition and retention. Learn. Mem. 10: 427-455.

Davidson, T.L. and Jarrard, L.E. 1993. A role for hippocampus in the utilization of hunger signals. Behav. Neural. Biol. 59: 167-171.

Disterhoft, J.F., Kronforst-Collins, M., Oh, M.M., Power, J.M., Preston, A.R., and Weiss, C. 1999. Cholinergic facilitation of trace eyeblink conditioning in aging rabbits. Life Sci. 64: 541-548.

Geinisman, Y., Disterhoft, J.F., Gundersen, H.J., McEchron, M.D., Persina, I.S., Power, J.M., van der Zee, E.A., and West, M.J. 2000. Remodeling of hippocampal synapses after hippocampus-dependent associative learning. J. Comp. Neurol. 417: 49-59.

Harker, K.T. and Whishaw, I.Q. 2004. Impaired place navigation in place and matching-to-place swimming pool tasks follows both retrosplenial cortex lesions and cingulum bundle lesions in rats. Hippocampus 14: 224-231.

Kronforst-Collins, M.A., Moriearty, P.L., Schmidt, B., and Disterhoft, J.F. 1997. Metrifonate improves associative learning and retention in aging rabbits. Behav. Neurosci. 111: 1031-1040.

Kuo, A.G., Lee, G., and Disterhoft, J.F. 2004. Alteration of sIAHP underlies reduction of AHP in rat CA1 pyramidal neurons after trace eyeblink conditioning. Soc. Neurosci. Abstr. 741: 13.

LoTurco, J.L., Coulter, D.A., and Alkon, D.L. 1988. Enhancement of synaptic potentials in rabbit CA1 pyramidal neurons following classical conditioning. Proc. Natl. Acad. Sci. 85: 1672-1676.
McEchron, M.D., Bouwmeester, H., Tseng, W., Weiss, C., and Disterhoft, J.F. 1998. Hippocampectomy disrupts auditory trace fear conditioning and contextual fear conditioning in the rat. Hippocampus 8: 638-646.

McNaughton, B.L., Barnes, C.A., Rao, G., Baldwin, J., and Rasmussen, M. 1986. Long-term enhancement of hippocampal synaptic transmission and the acquisition of spatial information. J. Neurosci. 6: $563-571$.

Morris, R.G., Garrud, P., Rawlins, J.N., and O'Keefe, J. 1982. Place navigation impaired in rats with hippocampal lesions. Nature 297: 681-683.

Moser, E.I., Moser, M.B., and Andersen, P. 1994. Potentiation of dentate synapses initiated by exploratory learning in rats: Dissociation from brain temperature, motor activity, and arousal. Learn. Mem. 1: $55-73$.

Moser, E.I., Krobert, K.A., Moser, M.B., and Morris, R.G. 1998. Impaired spatial learning after saturation of long-term potentiation. Science 281: 2038-2042.

Moyer Jr., J.R., Deyo, R.A., and Disterhoft, J.F. 1990. Hippocampectomy disrupts trace eye-blink conditioning in rabbits. Behav. Neurosci. 104: 243-252.

Moyer Jr., J.R., Thompson, L.T., and Disterhoft, J.F. 1996. Trace eyeblink conditioning increases CA1 excitability in a transient and learning-specific manner. J. Neurosci. 16: 5536-5546.

Nicholson, D.A., Yoshida, R., Berry, R.W., Gallagher, M., and Geinisman, Y. 2004. Reduction in size of perforated postsynaptic densities in hippocampal axospinous synapses and age-related spatial learning impairments. J. Neurosci. 24: 7648-7653.

Oh, M.M., Power, J.M., Thompson, L.T., Moriearty, P.L., and Disterhoft, J.F. 1999. Metrifonate increases neuronal excitability in CA1 pyramidal neurons from both young and aging rabbit hippocampus. J. Neurosci. 19: 1814-1823.

Oh, M.M., Kuo, A.G., Wu, W.W., Sametsky, E.A., and Disterhoft, J.F. 2003. Watermaze learning enhances excitability of CA1 pyramidal neurons. J. Neurophysiol. 90: 2171-2179.

Power, J.M., Thompson, L.T., Moyer Jr., J.R., and Disterhoft, J.F. 1997. Enhanced synaptic transmission in CA1 hippocampus after eyeblink conditioning. J. Neurophysiol. 78: 1184-1187.

Rioux, G.F. and Robinson, G.B. 1995. Hippocampal long-term potentiation does not affect either discrimination learning or reversal learning of the rabbit nictitating membrane response. Hippocampus 5: 165-170.

Robinson, G.B., Port, R.L., and Berger, T.W. 1989. Kindling facilitates acquisition of discriminative responding but disrupts reversal learning of the rabbit nictitating membrane response. Behav. Brain Res. 31: 279-283.

Saar, D., Grossman, Y., and Barkai, E. 1998. Reduced afterhyperpolarization in rat piriform cortex pyramidal neurons is associated with increased learning capability during operant conditioning. Eur. J. Neurosci. 10: 1518-1523.

Schwindt, P.C., Spain, W.J., Foehring, R.C., Stafstrom, C.E., Chubb, M.C., and Crill, W.E. 1988. Multiple potassium conductances and their functions in neurons from cat sensorimotor cortex in vitro. $J$. Neurophysiol. 59: 424-449.

Skelton, R.W., Scarth, A.S., Wilkie, D.M., Miller, J.J., and Phillips, A.G. 1987. Long-term increases in dentate granule cell responsivity accompany operant conditioning. J. Neurosci. 7: 3081-3087.

Steele, R.J. and Morris, R.G. 1999. Delay-dependent impairment of a matching-to-place task with chronic and intrahippocampal infusion of the NMDA-antagonist D-AP5. Hippocampus 9: 118-136.

Storm, J.F. 1990. Potassium currents in hippocampal pyramidal cells. Prog. Brain Res. 83: 161-187.

von Listow Roloff, E., Platt, B., and Riedel, G. 2002. Long-term study of chronic oral aluminum exposure and spatial working memory in rats. Behav. Neurosci. 116: 351-356.

Weiss, C., Bouwmeester, H., Power, J.M., and Disterhoft, J.F. 1999a. Hippocampal lesions prevent trace eyeblink conditioning in the freely moving rat. Behav. Brain Res. 99: 123-132.

Weiss, C., Knuttinen, M.G., Power, J.M., Patel, R.I., O'Connor, M.S., and Disterhoft, J.F. 1999b. Trace eyeblink conditioning in the freely moving rat: Optimizing the conditioning parameters. Behav. Neurosci. 113: 1100-1105.

Weiss, C., Preston, A.R., Oh, M.M., Schwarz, R.D., Welty, D., and Disterhoft, J.F. 2000. The M1 muscarinic agonist CI-1017 facilitates trace eyeblink conditioning in aging rabbits and increases the excitability of CA1 pyramidal neurons. J. Neurosci. 20: 783-790.

Weisz, D.J., Clark, G.A., and Thompson, R.F. 1984. Increased responsivity of dentate granule cells during nictitating membrane response conditioning in rabbit. Behav. Brain Res. 12: 145-154.

Received May 27, 2005; accepted in revised form December 8, 2005. 


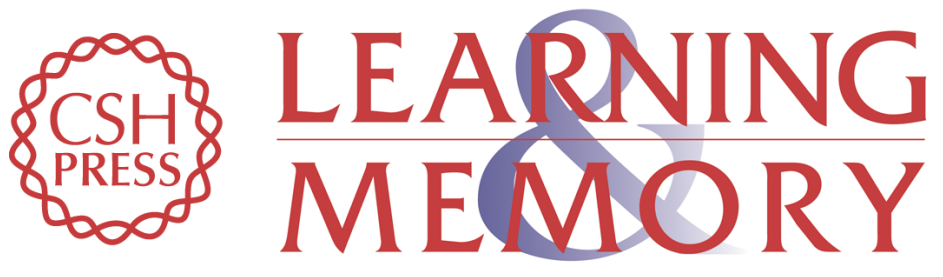

\section{Simultaneous training on two hippocampus-dependent tasks facilitates acquisition of trace eyeblink conditioning}

Amy G. Kuo, Grace Lee and John F. Disterhoft

Learn. Mem. 2006, 13:

Access the most recent version at doi:10.1101/lm.98406

References This article cites 36 articles, 13 of which can be accessed free at: http://learnmem.cshlp.org/content/13/2/201.full.html\#ref-list-1

License

Email Alerting Receive free email alerts when new articles cite this article - sign up in the box at the Service top right corner of the article or click here. 